\title{
Cost effectiveness of vaccination against pandemic influenza in European countries: mathematical modelling analysis
}

\author{
(a) (1) $\Theta$ OPEN ACCESS
}

\author{
Anna K Lugnér researcher ${ }^{1}$, Michiel van Boven senior researcher ${ }^{1}$, Robin de Vries researcher \\ pharmacoeconomics and access manager ${ }^{23}$, Maarten $\mathrm{J}$ Postma professor ${ }^{2}$, Jacco Wallinga senior \\ researcher and associate professor ${ }^{14}$
}

${ }^{1}$ Centre for Infectious Disease Control Netherlands, Epidemiology and Surveillance Unit, National Institute for Public Health and the Environment, PO Box 1, 3720 BA Bilthoven, Netherlands; ${ }^{2}$ Department of Pharmacy, Unit of PharmacoEpidemiology and PharmacoEconomics, University of Groningen, Groningen, Netherlands; ${ }^{3}$ Commercial Affairs, Roche Nederland, Woerden, Netherlands; ${ }^{4}$ University Medical Center Utrecht, Julius Center for Health Sciences and Primary Care, Utrecht, Netherlands

\begin{abstract}
Objective To investigate whether a single optimal vaccination strategy exists across countries to deal with a future influenza pandemic by comparing the cost effectiveness of different strategies in various pandemic scenarios for three European countries.

Design Economic and epidemic modelling study.

Settings General populations in Germany, the Netherlands, and the United Kingdom.

Data sources Country specific patterns of social contact and demographic data.

Model An age structured susceptible-exposed-infected-recovered transmission model that describes how an influenza A virus will spread in the populations of Germany, the Netherlands, and the United Kingdom.

Interventions Comparison of four vaccination strategies: no vaccination, blanket vaccination, vaccination of elderly people ( $\geq 65$ years), and vaccination of high transmitters (5-19 years). The four strategies were evaluated for scenarios in which a vaccine became available early or at the peak of the pandemic, and in which either everyone was initially susceptible or older age groups had pre-existing immunity.
\end{abstract}

Main outcome measure Cost per quality adjusted life years (QALYs) gained.

Results All vaccination strategies were cost effective (incremental cost per QALY gained, comparing intervention with non-intervention). In scenarios where the vaccine became available at the peak of the pandemic and there was pre-existing immunity among elderly people the incremental cost effectiveness ratios for vaccinating high transmitters were $€ 7325$ ( $£ 5815 ; \$ 10470$ ) per QALY gained for Germany, $€ 10216$ per QALY gained for the Netherlands, and $€ 7280$ per QALY gained for the United Kingdom. The most cost effective strategy not only differed across the pandemic scenarios but also between countries. Specifically, when the vaccine was available early in the pandemic and there was no pre-existing immunity, in Germany it would be most cost effective to vaccinate elderly people ( $€ 940$ per QALY gained), whereas it would be most cost effective to vaccinate high transmitters in both the Netherlands ( $€ 525$ per QALY gained) and the United Kingdom ( $€ 163$ per QALY gained). This difference in optimal strategies was due to differences in the demographic characteristics of the countries: Germany has a significantly higher proportion of elderly people compared with the Netherlands and the United Kingdom.

Conclusions No single vaccination strategy was most cost effective across countries. With aging populations, pre-existing immunity in particular could be of crucial importance for the cost effectiveness of options to mitigate a future influenza pandemic.

\section{Introduction}

Many countries have preparedness plans specifying how to deal with an influenza pandemic and in particular how to prioritise vaccination if the supply of vaccine falls short. For a small number of countries the proposed interventions in these plans have been tested in computer simulations using dynamic epidemiological models. ${ }^{1-6}$ For an even smaller number, the cost effectiveness of the proposed interventions has been analysed. ${ }^{7-9}$ 
Most countries adapt their plans from other countries or follow general advice from intergovernmental organisations such as the World Health Organization and the European Centre for Disease Prevention and Control. These organisations provide a wide range of countries with general advice on whether to vaccinate. Yet countries differ in their demographic characteristics, patterns of social contact, healthcare systems, and the cost structure of such systems. This raises the question of whether guidance should be tailored to the national level instead of providing a single recommendation supposedly suitable for a range of countries.

Vaccines against pandemic influenza can be produced, albeit in limited quantities, within a few months after isolation of the novel pandemic influenza strain. As the capacity for global production is limited, vaccine would be scarce. If a government wanted to purchase vaccine it would have to negotiate a contract with the manufacturers in the initial phase of the pandemic. A government would thus need to decide early on whether to purchase vaccines, the number of doses needed, and when to vaccinate. On the positive side, governments would have enough time between purchase and delivery to set up the infrastructure for a vaccination campaign. On the negative side, governments would need to make important decisions in the absence of accurate information and thus would need to allow for a variety of scenarios.

As a result of the scarcity of pandemic vaccines, decisions on how to allocate these vaccines optimally is important. When the economic impact of an influenza pandemic is included in the decision process, country specific details can be of great significance. Country specific demographic variables and patterns of social contact affect the transmission of influenza and may result in differences between the cost effectiveness of vaccines for countries with different demographic profiles. For example, in countries with a high percentage of older people without pre-existing immunity against a new influenza A virus strain it might be optimal to vaccinate just elderly people, whereas in countries with a low percentage of older people it might be optimal to vaccine young people, irrespective of the presence of pre-existing immunity in the population. If such situations are common, any general advice on a single best vaccination strategy might lose its importance.

We investigated whether a single vaccination strategy would be most cost effective in a range of different pandemic scenarios for different countries, which age group should be vaccinated to get the highest benefit from the resources spent, and whether different strategies apply in different countries. In particular we investigated the cost effectiveness of vaccination strategies in different pandemic scenarios for Germany, the Netherlands, and the United Kingdom; three countries that are geographically close and culturally similar but differ in their population structure.

\section{Methods}

We linked an age structured transmission model of pandemic influenza with healthcare consumption and unit costs for healthcare resources specific to Germany, the Netherlands, and the United Kingdom. This model incorporates demographic structures, distinguishes between groups at low and high risk for developing serious disease, and uses social contact patterns as a proxy for at risk contacts. The use of a dynamic model is essential as it enabled us to assess not only the direct effect of vaccination on those who are vaccinated (as in standard static models) but also the indirect effect because people who are not vaccinated are placed at a lower risk of infection.

\section{Model}

\section{Transmission model}

We used a deterministic age structured susceptible-exposed-infected-recovered epidemic model that describes how an influenza A virus would spread in a population. ${ }^{2}$ People were categorised into six age groups, preschool children (0-4 years), primary school children (5-12 years), secondary school children (13-19 years), young adults (20-39 years), older adults (40-64 years), and elderly people ( $\geq 65$ years), and classified as susceptible to infection, infected but not yet infectious, infected and infectious, or recovered, immune, or dead. The duration of the infection cycle was set to match an observed duration of the mean generation interval of 2.8 days for seasonal influenza ${ }^{10}$ and the novel pandemic A/H1N1 2009 influenza virus. ${ }^{11}$ This duration was obtained by setting the mean latent period at 1.95 days with a variance of 0.48 and by setting the mean infectious period at 1.6 days with a variance of 0.32 . We calibrated the transmissibility of the virus to render an overall basic reproduction ratio of 1.7 in a susceptible population, which is in line with epidemic growth rates observed in past influenza pandemics. ${ }^{10}$ As the typical timescale of an influenza pandemic wave is in the order of months, we did not include effects of changing demographics during the pandemic, such as aging and births, and deaths due to reasons other than influenza infection.

\section{Social contact patterns}

We calculated age specific contact patterns from data on self reported social contact rates for Germany, the Netherlands, and the United Kingdom in $2006 .{ }^{12}$ Such contacts were aggregated into the six age groups with similar contact characteristics. A summary of the contact rates for the six age categories shows that for all three countries, people tend to mix within their own age group (see supplementary table A.1).

\section{Demographic data}

We set the population size by age group as that for 2006, as obtained from official sources: Federal Statistical Office for Germany, Statistics Netherlands, and UK National Statistics (table $1 \Downarrow$ ).

\section{Low and high risk groups}

We divided each age group into two subgroups, one with people at relatively low risk and one with people at high risk of developing serious complications on infection. High risk groups included people who were immunocompromised, those with chronic respiratory diseases, and those aged over 65 in nursing homes. The share of the population in each age and risk group is available for the Netherlands. ${ }^{13}$ For the other two countries, information at this level of detail with comparable risk definitions was not available. In the absence of any evidence for large differences in risk groups between countries, we used the Dutch data for all three countries (table 1).

\section{Vaccination efficacy}

The vaccine was assumed to be an imperfect "all or nothing" vaccine, such that with a certain probability it would provide either perfect protection from infection or none at all (primary vaccine failure). For ages 0-64 years we set the vaccine efficacy (the probability that the vaccine would provide protection from infection) at $80 \%$. For those aged 65 or older, we assumed vaccine efficacy to be $56 \% .{ }^{14}$ We made no distinction for 
efficacy between people at high risk and low risk of serious complications.

\section{Evaluation of vaccination strategies in different scenarios}

\section{Vaccination strategies}

We investigated four alternative strategies for allocating the available vaccines over the various age groups: firstly, not to vaccinate; secondly, to offer vaccination to the whole population; thirdly to vaccinate elderly people; and finally, to vaccinate primary and secondary school children (ages 5-19), so called high transmitters because these age groups have relatively more contacts ${ }^{15}$ (see supplementary table A.1). Table $2 \Downarrow$ shows the percentages of vaccine uptake used for each of these strategies. Because for various reasons not all people eligible for vaccination would be vaccinated, we chose a vaccination coverage of at most $90 \%$ for everyone eligible for vaccination. Because pandemic influenza vaccine is not registered for infants under 6 months of age, the vaccination percentage is lower for the age group 0-4 years (table 2).

\section{Pandemic scenarios}

To detect whether there was a single best vaccination strategy for different countries under different circumstances, we evaluated the four alternative strategies for four plausible scenarios for an influenza pandemic.

In an optimistic scenario it is possible that a vaccine can be produced against the new virus strain and that a large number of vaccine doses would be available before the pandemic became established (early vaccination). In a more pessimistic scenario the same number of doses would become available during the pandemic and vaccination would take place at the peak of the pandemic (late vaccination). Simulations have shown that availability of vaccines two weeks before the peak of the pandemic or earlier give results similar to the early vaccination scenario, and availability of vaccines later than two weeks before the peak give results similar to the late vaccination scenario. ${ }^{2}$

Because an influenza pandemic is caused by a novel virus strain, it is possible that all those in the population are susceptible to infection with the new virus strain before vaccination starts, the so called "no immunity" scenario. As observations during the Asian influenza pandemic ${ }^{16}$ and the recent pandemic in $2009^{17}$ have shown, it is also possible for older people to have partial cross immunity to the novel virus strain. We termed the scenarios where some older people are protected from infection at the start of the pandemic and before vaccination starts as "pre-existing immunity." The precise values of variables and parameters in each of these scenarios are based on serological studies during the 2009 influenza pandemic (table $3 \Downarrow$ ).

\section{Cost effectiveness}

The cost per quality adjusted life year (QALY) gained was calculated as the incremental cost effectiveness ratio of the specific intervention strategy compared with the non-intervention option. The incremental cost effectiveness ratio represents the additional cost of one QALY gained and is calculated as the difference in cost divided by the difference in QALYs. A lower value for the incremental cost effectiveness ratio indicates a more cost effective intervention. Often there is no official threshold for when an intervention is considered to be cost effective. In the United Kingdom, the National Institute for Health and Clinical Excellence suggests a threshold range of $£ 20000$ ( $€ 24786$; $\$ 31400$ ) to $£ 30000 .{ }^{18}$ Here we apply the higher of these (representing €37 800) for both the United Kingdom and Germany. For the Netherlands no official threshold exists, but $€ 20000$ is mostly cited. ${ }^{19}{ }^{20}$.

\section{Healthcare use}

It was assumed that $60 \%$ of the infected people developed influenza-like illness and that the rest had no symptoms. ${ }^{21}{ }^{22} \mathrm{~A}$ proportion $(25 \%)$ of those with symptoms seek medical help. Several types of healthcare consumption were included in the calculations: over-the-counter drug use, visits to a general practitioner, prescription drugs (antibiotics to prevent or treat secondary infections), and admissions to hospital in normal wards $(85 \%)$ and intensive care wards $(15 \%) .{ }^{13}$ We assumed that people with influenza-like illness received antiviral drugs if in contact with a general practitioner, but we did not assume any influence on transmission or complication rates. Rates of hospital admission were based on Dutch data and were applied to all three countries (see supplementary table A.2). ${ }^{23}$

\section{Costs}

From the published literature we obtained country specific data on cost of using healthcare resources (direct costs) and percentages of cases of influenza-like illness using the healthcare resources (table $4 \Downarrow$ ). When we could find no specific data for Germany or the United Kingdom, we took data available for the Netherlands as a proxy. This was the case for the direct cost estimates of the percentages seeking medical help for influenza-like illness, prescribed antibiotics during a visit to their doctor, using over-the-counter drugs, and admitted to intensive care. Some variation may be expected in these proportions between the countries. However, the expectation is that such variation would not be large enough to influence the cost effectiveness ratios substantially. Vaccine costs are mainly unknown and were based on unofficial sources. We assumed a two dose schedule, and it was the same for all three countries. According to the Dutch guidelines, costs for production losses should be included in cost effectiveness analysis and estimated using the friction costing approach. ${ }^{24}$ In the German guidelines, the analyst's perspective determines whether to include production losses. ${ }^{25}$ According to the UK guidelines, all important items should be included, and the inclusion of production losses is not always required. ${ }^{26}$ We calculated all costs with and without inclusion of production losses. For all cases of influenza-like illness we used the friction cost method for the Netherlands and the human capital approach for the other two countries. The main differences between the two methods is that the human capital approach estimates a potential value of lost production due to absenteeism or death, whereas the friction cost method suggests that the production losses might be smaller. Owing to replacement of long term absent (or deceased) employees and the assumption that short term absenteeism will be partly caught up with when sick employees return to work, absent employees contribute to the production losses only during the "friction period" until they are replaced. ${ }^{27}$ The difference between the magnitude of production losses estimated with the two methods can be considerable when estimating production losses due to deaths. To avoid the exaggeration of differences in production losses between countries owing to differences in methodology, we estimated production losses due to deaths for all countries using the friction cost method.

We recalculated all costs to reflect price levels in 2008. These were expressed in euros using the average exchange rate between the euro and the pound sterling over the year 2008. Costs were 
not discounted owing to the relatively short time perspective of the analysis (within one year) on resource and financial impacts.

\section{Quality of life and life years lost}

Losses in quality of life due to an influenza episode were based on burden of disease estimates for the Netherlands ${ }^{28}$ for the scenario of no immunity. During the 2009 pandemic, estimates of quality of life were gathered in the United Kingdom. ${ }^{7} \mathrm{We}$ used these weights for the scenario of pre-existing immunity, reflecting the burden of illness due to the 2009 pandemic virus. For cases admitted to hospital, the utility losses were 2.17 times higher than for influenza-like illness ${ }^{7}$ (table $5 \Downarrow$ ).

To estimate QALYs gained we based the remaining, age specific, life years used in the economic calculations on country specific demographic predictions for 2006 (table 1). These were calculated with a weighting factor for the high and low risk groups. The low risk group was weighted with a factor of 1.15 and the high risk group with a factor of 0.75 , reflecting a longer expected remaining lifetime for a healthy individual compared with someone with chronic conditions. ${ }^{29}$ We used the same case fatality rate for all three countries, estimated as three age group specific excess death rates due to influenza (see supplementary table A.2).$^{30}$ For the economic evaluation, we discounted life years at country specific rates, according to the respective guidelines: Germany at $5 \%$, the Netherlands at $1.5 \%$, and the United Kingdom at $3.5 \%$.

\section{Sensitivity analysis}

We explored the sensitivity of the health economic outcome for uncertainty about the precise value of epidemic and economic variables. Just as it is common practice to report the outcome of data analysis as a point estimate with confidence intervals to indicate the precision of this estimate, it is common to report the projected incremental cost effectiveness ratios for a future pandemic with a sensitivity analysis indicating the robustness of this projection. We focused on five key variables.

The first key variable was the cost of the vaccine. We chose a default value for unit costs of $€ 10$ and assessed the sensitivity of the health economic outcome of doubling the unit cost of the vaccine to $€ 20$, so called high costs. Other variables that affect the costs of intervention would result in a similar sensitivity as this cost of vaccination. The second key variable was the discount rate for the life years gained. We lowered the discount rates from the prescribed value for each country to zero (no discounting). As a change in the discount rates results in a different weighting of the number of life years gained, it also assesses the sensitivity variables that affect the number of life years gained.

The third key variable was the basic reproduction ratio, which incorporates the effects of variations in transmissibility and infectious period in one measure. We varied the basic reproduction ratio from a default value of 1.7 to 1.55 (low transmissibility) and 1.85 (high transmissibility). These low and high values represented the two midpoints between the default value, which we deemed most plausible, and the most extreme values that are still consistent with observations of influenza pandemics. ${ }^{2531}$ The fourth key variable was vaccination coverage. We varied the coverage from values in the base case for each strategy (table 2) to half of these values (low vaccination coverage). The fifth key variable was the level of pre-existing immunity. We varied the level from values in the base case (table 3 ) to a half higher than the base case (high pre-existing immunity). Taken together, these epidemiological variables span possible pandemics.
To explore potential interactions between the epidemiological variables and health economic variables, we assessed the six combinations of values for the basic reproduction ratio (1.55, 1.7 , and 1.8$)$ and vaccine costs ( $€ 10$ unit costs, $€ 20$ unit costs).

\section{Results}

The clinical attack rate for an uncontrolled influenza pandemic is similar for Germany, the Netherlands, and the United Kingdom. The scenario where the total population is assumed to be fully susceptible results in an overall clinical attack rate of about $36 \%$. When assuming pre-existing immunity in part of the population, the overall clinical attack rate is about $27 \%$.

Table $6 \Downarrow$ shows the costs of vaccination, the difference in direct healthcare costs (net direct costs), the difference in cost including productivity losses (net total costs), and the QALY gained for each vaccine availability for the different strategies. The incremental cost effectiveness ratios for the vaccination strategies are below country specific thresholds for all scenarios for all three countries, and therefore all the interventions were cost effective. For all three countries, all vaccination strategies were more cost effective (that is, the incremental cost effectiveness ratio was lower) in scenarios with no immunity compared with scenarios with pre-existing immunity (table 6).

If production losses were included in the health economic analysis, most vaccination strategies were cost saving for all three countries. There were a few exceptions: in scenarios with pre-existing immunity and in the scenario without immunity and the vaccine being available late in the pandemic, vaccinating elderly people was not cost saving for all three countries; in the scenarios where the vaccine became available late in the epidemic, vaccinating the whole population would not be cost saving in the United Kingdom but would be in Germany and the Netherlands.

In summary, the most cost effective vaccination strategy depended on both the pandemic scenario and the country (table $7 \Downarrow$ ). For instance, in the scenario without immunity and the vaccine being available early in the pandemic, vaccinating elderly people was the most cost effective strategy for Germany ( 940 per QALY gained), but vaccinating young people at school (the high transmitters) was the most cost effective strategy for the Netherlands ( $€ 525$ per QALY gained) and the United Kingdom ( $€ 163$ per QALY gained, table 6$)$.

To investigate why it would be most cost effective to vaccinate young people at school rather than elderly people, two vaccination strategies were looked at in more detail (vaccinating elderly people and vaccinating high transmitters) for the Netherlands and Germany, under the no immunity scenario. The costs of vaccination and cost savings due to vaccination (net direct costs) attributed to each age group as well as the QALYs gained in that age group were calculated. The figure $\Downarrow$ shows the results (costs per 10000 population and QALYs gained per 100000 population). For the Netherlands, when high transmitters were vaccinated the costs of vaccinating the age groups 5-12 and 13-19 were higher than the costs saved in those age groups, but because transmission was reduced the cost savings and QALY gains in other age groups were large. The cost savings in all groups, when taken together, were large enough to make this the most cost effective strategy. This was not so for Germany, where the costs saved and QALYs gained in the age groups older than 20 did not compensate for the costs in the vaccinated age groups (5-12 and 13-19). Compared with the Netherlands it would be more cost effective for Germany to vaccinate the older age group, where relatively more QALYs 
were gained. This difference remained when discounting was omitted.

To assess the robustness of findings the key variables in the analyses were varied (table $8 \Downarrow$ ). In most of these analyses (with the exception of Germany), vaccinating high transmitters was the most cost effective option. With a low vaccination coverage (half of the assumption in the base case) vaccinating the whole population would be the most cost effective strategy for Germany.

The influence of the relatively high discount rate used for the QALYs in Germany on the incremental cost effectiveness ratios (no immunity and early availability of vaccine) were investigated. The incremental cost effectiveness ratio of vaccinating high transmitters was about $9.4 \%$ higher than that of vaccinating elderly people when the QALYs were not discounted. When QALYs were discounted, the incremental cost effectiveness ratio of vaccinating high transmitters was about $8.8 \%$ higher than that of elderly people (comparing results in table 6 with the sensitivity analysis "no discounting" reported in supplementary table A.3). This suggests that the discount rate does not determine the finding that vaccinating elderly people first would be the most cost effective option.

Doubling the costs of the vaccine for each scenario did not change the most cost effective strategy, and the most cost effective strategy remained cost effective. Changing the basic reproduction ratio to a lower value resulted in smaller epidemics. This resulted in a few changes in the best vaccination strategy - for example, in the scenario with early availability of vaccine without pre-existing immunity, the most cost effective strategy for Germany shifted from vaccinating elderly people towards vaccinating high transmitters. Changing the basic reproduction ratio to a higher value resulted in larger epidemics. In the scenario with late availability of vaccine and pre-existing immunity, the most cost effective strategy shifted for all three countries from vaccinating high transmitters towards vaccinating elderly people.

When the results of the sensitivity analyses were compared with the base case including the productivity losses, the strategies were no longer cost saving in several scenarios (see supplementary tables A.3-A.5). For example, with higher vaccine costs, vaccinating the whole population in Germany in the late stage was no longer cost saving. In general, with more pre-existing immunity all vaccination strategies became less cost effective.

This suggests that the finding that the most cost effective vaccination strategy depended on both the pandemic scenario and the country was robust to the particular choice of variable values.

\section{Discussion}

In this study we evaluated the cost effectiveness of four vaccination strategies in Germany, the Netherlands, and the United Kingdom, to find out which would be the most cost effective for different influenza pandemic scenarios and if that strategy differed among countries. The scenarios were no vaccination, vaccinating elderly people, vaccinating high transmitters, and vaccinating the whole population. The four pandemic influenza scenarios were no immunity and vaccine available either early or late and pre-existing immunity and vaccine available either early or late. We found that the most cost effective strategy differed between pandemic scenarios and between countries. At the population level, all vaccination strategies were cost effective (calculated as incremental cost per QALY gained, comparing vaccination with no vaccination) taking the country specific thresholds into account.

For all scenarios we found that vaccinating the whole population was a suboptimal cost effective strategy. The most cost effective strategy depended on both the scenario and the country. For most scenarios we found that it would be most cost effective to vaccinate young people aged 5-19 years (high transmitters). The exceptions were that vaccinating elderly people was most effective in a scenario without immunity and with the vaccine becoming available late in the pandemic, in all countries. In Germany, vaccinating elderly people would still be the most cost effective strategy in the scenario without immunity and when vaccine became available early in the pandemic. A possible explanation for the discrepancy between optimal strategies for Germany and the other two countries lies in the age profile of the population. In Germany about $20 \%$ of the population is 65 years or older, compared with about $14 \%$ in the Netherlands and 16\% in the United Kingdom. In general, in the elderly age group a higher proportion are at a high risk of complications.

We tested whether differences in demography can account for the discrepancy in optimal vaccination strategies. We re-evaluated the vaccination strategies for Germany with the age profile of the German population replaced by that of the Dutch population. For the scenario where vaccine was available early and there was no pre-existing immunity, the most cost effective strategy changed from vaccinating elderly people to vaccinating high transmitters,. This confirms that the age profile of the population can explain the observed discrepancy between countries. We also evaluated the vaccination strategies for Germany where we changed the cost structure of Germany to that of the Netherlands. Again we found that the most cost effective strategy changed from vaccinating elderly people to vaccinating high transmitters. This stresses the need for country specific analyses to determine the most cost effective vaccination strategy, explicitly accounting for the country specific demography and cost structure.

\section{Strengths and weaknesses of the study}

So far, no pandemic preparedness models of the cost effectiveness of different vaccination strategies have incorporated pre-existing immunity in elderly people or other age groups. Since pre-existing immunity has played an important part in the recent 2009 pandemic, we incorporated it in a scenario for a future pandemic influenza virus strain. It might have been expected that the role of pre-existing immunity would make all vaccination strategies less cost effective. Here we showed that pre-existing immunity leads to the strategy of vaccinating high transmitters being optimal. It might also be expected that the absence of pre-existing immunity would have a larger impact on the cost effectiveness in a country where the proportion of people in the older age groups is high, such as in Germany. Indeed here we showed that for a population with a high proportion of older people, in the absence of pre-existing immunity, the strategy of vaccinating elderly people was optimal.

Not all data were available for all three countries. We chose to be conservative in our comparison between the countries and to use the same data for all three countries. We used the data from the Netherlands for the risk of being admitted to hospital, the risk of death due to infection, and the distribution in risk groups. Despite the conservative choices, we found that the existing differences between the demographic characteristics, 
especially the proportion of elderly people, suffice to result in different optimal vaccination strategies between the countries.

\section{Strengths and weaknesses in relation to other studies}

We did not attempt to calibrate the transmission model to the recent pandemic A/H1N1 2009 influenza, as we did not seek to generate the exact outcomes registered for this pandemic in one specific country but rather to present a general analysis of which strategy would be most cost effective in a future pandemic. Vaccinating against the recent pandemic in the United States showed that it was cost effective for several different attack rates and for different age and risk groups according to the estimations carried out using a decision analytical model. ${ }^{32}$ Some economic evaluations of vaccination strategies have also been done during the recent pandemic using dynamic models. In Ontario, Canada it was assumed that the vaccination coverage was $30 \%$ within the population. It was shown that the vaccination strategy was cost effective $(<\$ C 5000$ per QALY gained). ${ }^{33}$ In the United Kingdom, a transmission model was fitted to the data from the recent pandemic. In that study, a probabilistic economic model showed a high probability that vaccinating high risk groups was cost effective; $98 \%$ of the simulations resulted in an incremental cost effectiveness ratio that was less than $£ 30000$ per QALY gained. ${ }^{7}$ Note that in these simulations of the $\mathrm{A} / \mathrm{H} 1 \mathrm{~N} 1$ pandemic the cost of the vaccine was not included but instead regarded as a sunk cost. In our analyses we included the cost of vaccination for a future pandemic and hence the results of the health economic analyses of the recent pandemic cannot be directly compared with our health economic analyses of expected future pandemics.

Our analysis shows that the use of dynamic modelling is crucial when studying a transmissible disease such as influenza. An earlier study to determine the optimal strategy for distributing a vaccine against a pandemic influenza virus used a static model that did not take transmission into account. ${ }^{34}$ The option of vaccinating children and young adults was then not even considered. When vaccinating young people, the intervention may not be cost effective if only the costs and effects in that group are looked at, as we have shown (figure). Including effects and cost savings at the population level changes the cost effectiveness of the intervention, since other age groups benefit from reduced transmission. We have added to the understanding of this indirect benefit and shown that, in particular, it influences cost effectiveness of pandemic vaccination strategies. This is important information in making decisions on how to control or mitigate an influenza pandemic.

\section{Implications of study findings}

Our primary objective was to compare the cost effectiveness of vaccination strategies against an influenza pandemic for countries with a similar cultural background, to examine the usefulness of general advice on a vaccination strategy.

Since, compared with no vaccination, almost all vaccination strategies were cost effective in almost all scenarios, the choice of cost effective vaccination strategies is ample. Next to considerations of cost effectiveness, decision makers should factor in many other aspects, ranging from ethical considerations to practical implementations. For example, vaccinating elderly people is an attractive strategy since an existing infrastructure for delivering seasonal influenza vaccination programmes can be used to reach the target groups, whereas vaccinating high transmitters requires a new infrastructure to reach this target group, and the willingness to vaccinate might be lower among these younger age groups.

We expect that the results may still hold even when decisions are based on objectives other than maximising cost effectiveness. This is relevant because a government also could choose to minimise the number of infections, deaths, life years lost, or the peak healthcare demand. Whereas in general a different objective will result in a different optimal strategy, many simulations have shown that in the control of infectious diseases different objectives can result in similar optimal allocation patterns of scarce vaccines. ${ }^{15} 35$ The intuition behind this result is that a strategy that will break the transmission chain with the minimum of effort will simultaneously minimise the number of infections, deaths, life years lost, or peak healthcare demand and will achieve a high cost effectiveness of infection control. Here, vaccination of high transmitters usually is the strategy that could break the transmission chain with minimal efforts-that is, vaccine doses administered.

\section{Unanswered questions and future research}

We have not dealt with the ethical issues involved in vaccinating younger age groups to protect older age groups, and we have not tackled the risk of adverse events from vaccination. The possible relation between the pandemic vaccine and the occurrence of narcolepsy ${ }^{36}{ }^{37}$ highlights that the ethical aspects of a strategy where one group in the population carries the potential risks of vaccination to protect another part of the population should also be taken into consideration.

\section{Conclusion}

According to our analysis, no single vaccination strategy is most cost effective across countries. There are, however, some general rules. In most but not all scenarios, not vaccinating was the worst strategy and vaccinating young people aged 5-19 years was the most cost effective. Exceptions to this rule occurred when vaccine became available early in the pandemic and there was pre-existing immunity: depending on the proportion of elderly people in the population, vaccinating elderly people was the best strategy. These findings are of crucial importance for the cost effectiveness of options to mitigate a future influenza pandemic. We conclude that a general recommended vaccination strategy should be considered with due caution. It makes sense to advise about alternative strategies and to suggest preference for one over another according to the particular country. One such factor that countries may heed is the proportion of elderly people in the population, which seems to determine which strategy performs best.

We thank Jan van de Kassteele for calculating the contact matrices and the reviewers (A Fischer and I Hall) for their constructive comments.

Contributors: AKL, MvB, MJP, and JW designed and conceptualised the study. $A K L$ and $M v B$, analysed the data and drafted the manuscript. $A K L, M v B, M J P$, and JW interpreted all data and analyses and revised the manuscript. AKL and RdV collected and interpreted the study data. AKL, MJP, and JW are the guarantors.

Funding: This research was partly funded by a Quantitative Immunization and Vaccine-Related Research (QUIVER) grant from the World Health Organization. The study sponsor had no influence on the study design; collection, analysis and interpretation of data; in the writing of the report; and in the decision to submit the article for publication.

Competing interests: All authors have completed the ICMJE uniform disclosure form at www.icmje.org/coi_disclosure.pdf (available on request from the corresponding author) and declare that: no support from any organisation for the submitted work; RdV is an employee of 


\section{What is already known on this topic}

Many countries have preparedness plans for pandemic influenza, specifying how vaccination should be prioritised if vaccine supply falls short

Most countries have adapted their plans from other countries or from intergovernmental organisations such as WHO

Countries differ by demography, social contact patterns, healthcare systems, and cost structure of healthcare, and it is not known how this affects the most cost effective strategy to prioritise pandemic influenza vaccine

\section{What this study adds}

Based on a mathematical model, the most cost effective strategy for pandemic influenza vaccination in Germany, the Netherlands, and the United Kingdom would, with few exceptions, be to prioritise 5-19 year olds, the high transmitter group

With no pre-existing immunity and early vaccination, the optimal strategy differed between countries and was determined by the proportion of elderly people in the population

General recommendations of a single strategy for a range of similar countries on how to prioritise pandemic influenza vaccines should be considered cautiously

Roche Nederland (since completing this paper) that might have an interest in the submitted work in the previous three years, MJP has been on advisory boards of, or received grants from Pfizer, GlaxoSmithKline, Sanofi Pasteur MSD, and MapiValues, that might have an interest in the submitted work in the previous three years; and no other relationships or activities that could appear to have influenced the submitted work.

\section{Ethical approval: Not required.}

Data sharing: The model code and dataset are available on request from the corresponding author anna.lugner@rivm.nl.

Longini IM Jr, Halloran ME, Nizam A, Yang Y. Containing pandemic influenza with antiviral agents. Am J Epidemiol 2004;159:623-33.

2 Mylius SD, Hagenaars TJ, Lugner AK, Wallinga J. Optimal allocation of pandemic influenza vaccine depends on age, risk and timing. Vaccine 2008;26:3742-9.

3 Longini IM Jr, Nizam A, Xu S, Ungchusak K, Hanshaoworakul W, Cummings DA, et al. Containing pandemic influenza at the source. Science 2005;309:1083-7.

4 Germann TC, Kadau K, Longini IM Jr, Macken CA. Mitigation strategies for pandemic influenza in the United States. Proc Natl Acad Sci USA 2006;103:5935-40.

5 Ferguson NM, Cummings DA, Fraser C, Cajka JC, Cooley PC, Burke DS. Strategies for mitigating an influenza pandemic. Nature 2006;442:448-52.

6 Ferguson NM, Cummings DA, Cauchemez S, Fraser C, Riley S, Meeyai A, et al. Strategies for containing an emerging influenza pandemic in Southeast Asia. Nature 2005;437:209-14

7 Baguelin M, Hoek AJ, Jit M, Flasche S, White PJ, Edmunds WJ. Vaccination against pandemic influenza $\mathrm{A} / \mathrm{H} 1 \mathrm{~N} 1 \mathrm{v}$ in England: a real-time economic evaluation. Vaccine 2010;28:2370-84.

8 Lugner AK, Mylius SD, Wallinga J. Dynamic versus static models in cost-effectiveness analyses of anti-viral drug therapy to mitigate an influenza pandemic. Health Econ 2010;19:518-31.

9 Sander B, Nizam A, Garrison LP Jr, Postma MJ, Halloran ME, Longini IM Jr. Economic evaluation of influenza pandemic mitigation strategies in the United States using a stochastic microsimulation transmission model. Value Health 2009;12:226-33.

10 Wallinga J, Lipsitch M. How generation intervals shape the relationship between growth rates and reproductive numbers. Proc Biol Sci 2007:274:599-604.

11 Hahne S, Donker T, Meijer A, Timen A, van Steenbergen J, Osterhaus A, et al. Epidemiology and control of influenza $A(H 1 N 1) v$ in the Netherlands: the first 115 cases. Euro Surveill 2009;14(27).

12 Mossong J, Hens N, Jit M, Beutels P, Auranen K, Mikolajczyk R, et al. Social contacts and mixing patterns relevant to the spread of infectious diseases. PLOS Med 2008:5:e74.

13 Van Genugten ML, Heijnen ML, Jager JC. Pandemic influenza and healthcare demand in the Netherlands: scenario analysis. Emerg Infect Dis 2003:9:531-8.

14 Gross PA, Hermogenes AW, Sacks HS, Lau J, Levandowski RA. The efficacy of influenza vaccine in elderly persons. A meta-analysis and review of the literature. Ann Intern Med 1995;123:518-27.

15 Wallinga J, van Boven M, Lipsitch M. Optimizing infectious disease interventions during an emerging epidemic. Proc Natl Acad Sci USA 2010;107:923-8.

16 Mulder J, Masurel N. Pre-epidemic antibody against 1957 strain of Asiatic influenza in serum of older people living in the Netherlands. Lancet 1958;1:810-4.

17 Miller M Viboud C, Simonsen L, Olson DR, Russell C. Mortality and morbidity burden associated with $\mathrm{A} / \mathrm{H} 1 \mathrm{~N} 1 \mathrm{pdm}$ influenza virus. Who is likely to be infected, experience clinical symptoms or die from the H1N1pdm 2009 pandemic virus? PLoS Currents: Influenza 2009;RRN1013.

18 NICE. Social value judgements: principles for the development of NICE guidance.

19 Casparie AF, van Hout BA, Simoons ML. [Guidelines and costs]. Ned Tijdschr Geneeskd 1998;142:2075-7.

20 Boersma C, Broere A, Postma MJ. Quantification of the potential impact of cost-effectiveness thresholds on Dutch drug expenditures using retrospective analysis. Value Health 2010;13:853-6.

21 Fukuda K Levandowski RA, Bridges CB, Cox NJ, eds. Inactivated influenza vaccines. Saunders, 2004

22 Jordan WS Jr, Denny FW Jr, Badger GF, Curtiss C, Dingle JH, Oseasohn R, et al. A study of illness in a group of Cleveland families. XVII. The occurrence of Asian influenza. Am J Hyg 1958;68:190-212.
23 Baltussen RM, Reinders A, Sprenger MJ, Postma MJ, Jager JC, Ament AJ, et al. Estimating influenza-related hospitalization in The Netherlands. Epidemiol Infect 1998;121:129-38.

24 Oostenbrink JB Bouwmans CAM, Koopmanschap MA, Rutten FFH. Handleiding voor kostenonderzoek, methoden en standaard kostprijzen voor economische evaluaties in de gezondheidszorg. Geactualiseerde versie 2004. [In Dutch]. Diemen: College voor zorgverzekeringen, 2004

25 ISPOR. International Society for Pharmacoeconomics and Outcomes Research. 2009 www.ispor.org.

26 National Institute for Health and Clinical Excellence. The guidelines manual 2009. NICE, 2009.

27 Koopmanschap MA, Rutten FF, van Ineveld BM, van Roijen L. The friction cost method for measuring indirect costs of disease. J Health Econ 1995;14:171-89.

28 Melse JM, Essink-Bot ML, Kramers PG, Hoeymans N. A national burden of disease calculation: Dutch disability-adjusted life-years. Dutch Burden of Disease Group. Am J Public Health 2000;90:1241-7.

29 Postma MJ, Bos JM, van Gennep M, Jager JC, Baltussen R, Sprenger MJ. Economic evaluation of influenza vaccination. Assessment for The Netherlands. Pharmacoeconomics 1999;16(Suppl 1):33-40.

30 Sprenger MJ, Mulder PG, Beyer WE, Van Strik R, Masurel N. Impact of influenza on mortality in relation to age and underlying disease, 1967-1989. Int $J$ Epidemiol 1993;22:334-40.

31 Boelle PY, Ansart S, Cori A, Valleron AJ. Transmission parameters of the A/H1N1 (2009) influenza virus pandemic: a review. Influenza Other Respi Viruses 2011;5:306-16.

32 Prosser LA, Lavelle TA, Fiore AE, Bridges CB, Reed C, Jain S, et al. Cost-effectiveness of 2009 pandemic influenza $A(H 1 N 1)$ vaccination in the United States. PLoS One 2011;6:e22308.

33 Sander B, Bauch CT, Fisman D, Fowler RA, Kwong JC, Maetzel A, et al. Is a mass immunization program for pandemic (H1N1) 2009 good value for money? Evidence from the Canadian Experience. Vaccine 2010;28:6210-20.

34 Meltzer MI, Cox NJ, Fukuda K. The economic impact of pandemic influenza in the United States: priorities for intervention. Emerg Infect Dis 1999;5:659-71.

35 Medlock J, Galvani AP. Optimizing influenza vaccine distribution. Science 2009;325:1705-8.

36 Nohynek H, Jokinen J, Partinen M, Vaarala O, Kirjavainen T, Sundman J, et al. AS03 adjuvanted $\mathrm{AH} 1 \mathrm{~N} 1$ vaccine associated with an abrupt increase in the incidence of childhood narcolepsy in Finland. PLoS One 2012;7:e33536.

37 Partinen M, Saarenpää-Heikkilä O, Ilveskoski I, Hublin C, Linna M, Olsén P, et al. Increased incidence and clinical picture of childhood narcolepsy following the $2009 \mathrm{H} 1 \mathrm{~N} 1$ pandemic vaccination campaign in Finland. PLoS One 2012;7:e33723.

38 StatistischeBundesamtDeutschland. Statistische Bundesamt Deutschland. 2009. www. genesis.destatis.de.

39 CBS. Statistics Netherlands: Statline. 2009. http://statline.cbs.nl/statweb/.

40 Governments Actuary's Department, United Kingdom. 2009. www.gad.gov.uk

41 Aballea S, Chancellor J, Martin M, Wutzler P, Carrat F, Gasparini R, et al. The cost-effectiveness of influenza vaccination for people aged 50 to 64 years: an international model. Value Health 200710:98-116.

42 Postma MJ, Jansema P, Scheijbeler HW, van Genugten ML. Scenarios on costs and savings of influenza treatment and prevention for Dutch healthy working adults. Vaccine 2005;23:5365-71.

43 Sander B, Hayden FG, Gyldmark M, Garrison LP Jr. Post-exposure influenza prophylaxis with oseltamivir: cost effectiveness and cost utility in families in the UK. Pharmacoeconomics 2006;24:373-86

44 Medicijnkosten. Drug costs. [ln Dutch]. www.medicijnkosten.nl.

45 Wallinga J, Teunis P, Kretzschmar M. Using data on social contacts to estimate age-specific transmission parameters for respiratory-spread infectious agents. $A m J$ Epidemiol 2006;164:936-44.

\section{Accepted: 25 May 2012}

\section{Cite this as: BMJ 2012;345:e4445}

This is an open-access article distributed under the terms of the Creative Commons Attribution Non-commercial License, which permits use, distribution, and reproduction in any medium, provided the original work is properly cited, the use is non commercial and is otherwise in compliance with the license. See: http://creativecommons.org/licenses/by$\mathrm{nc} / 2.0 /$ and http://creativecommons.org/licenses/by-nc/2.0/legalcode. 


\section{Tables}

Table 1/ Demographic data for Germany, Netherlands, and United Kingdom by age group

\begin{tabular}{|c|c|c|c|c|c|c|}
\hline \multirow[b]{2}{*}{ Variables } & \multicolumn{6}{|c|}{ Age group (years) } \\
\hline & $0-4$ & $5-12$ & $13-19$ & $20-39$ & $40-64$ & $\geq 65$ \\
\hline \multicolumn{7}{|l|}{ Germany: } \\
\hline$\%$ of population* & 4 & 9 & 7 & 25 & 35 & 20 \\
\hline$\%$ of population in high risk group $\dagger$ & 0.006 & 0.020 & 0.014 & 0.412 & 0.754 & 1.00 \\
\hline Remaining life yearsł & 77.6 & 71.3 & 63.8 & 50.6 & 29.6 & 8.1 \\
\hline \multicolumn{7}{|l|}{ Netherlands: } \\
\hline$\%$ of population* & 6 & 11 & 7 & 26 & 35 & 14 \\
\hline$\%$ of population in high risk group $\dagger$ & 0.008 & 0.025 & 0.015 & 0.428 & 0.751 & 0.733 \\
\hline Remaining life years $\ddagger$ & 78.4 & 72.2 & 64.8 & 51.5 & 30.2 & 8.4 \\
\hline \multicolumn{7}{|l|}{ United Kingdom: } \\
\hline$\%$ of population* & 6 & 11 & 8 & 27 & 32 & 16 \\
\hline$\%$ of population in high risk group $\dagger$ & 0.008 & 0.025 & 0.016 & 0.442 & 0.697 & 0.809 \\
\hline Remaining life years $\ddagger$ & 88.9 & 81.7 & 73.3 & 58.3 & 36.3 & 10.4 \\
\hline
\end{tabular}

*Total population: Germany 82314 906; Netherlands 16357 992; United Kingdom 60587800.

†Age group specific shares of population in high risk group based on Dutch data. ${ }^{13}$

fUndiscounted projections from 2006, here calculated as averages in age group. ${ }^{39} 40$ 
Table 2| Percentages of population vaccinated by age group and vaccination strategy

\begin{tabular}{|c|c|}
\hline & Age group (years) \\
\hline Scenarios & $\begin{array}{lllll}0-4 & 5-12 & 13-19 & 20-39 & 40-64 \geq 65\end{array}$ \\
\hline
\end{tabular}

\begin{tabular}{lllllll} 
No vaccination & 0 & 0 & 0 & 0 & 0 & 0 \\
\hline
\end{tabular}

Whole population $\begin{array}{llllll}54 & 90 & 90 & 90 & 90 & 90\end{array}$

\begin{tabular}{lcccccc}
\hline Elderly people & 0 & 0 & 0 & 0 & 0 & 90 \\
\hline High transmitters & 0 & 90 & 90 & 0 & 0 & 0
\end{tabular}


Table 3| Description of four scenarios for influenza pandemic

\begin{tabular}{lccccccc} 
& \multicolumn{7}{l}{ Proportion immune at start of pandemic by age group } \\
\cline { 2 - 7 } Scenarios & $\mathbf{0 - 4}$ & $\mathbf{5 - 1 2}$ & $\mathbf{1 3 - 1 9}$ & $\mathbf{2 0 - 3 9}$ & $\mathbf{4 0 - 6 4}$ & $\mathbf{2 6 5}$ \\
$\begin{array}{l}\text { Early vaccination*: } \\
\text { No immunity }\end{array}$ & 0 & 0 & 0 & 0 & 0 & 0 \\
\hline Pre-existing immunity & 0 & 0 & 0 & 0 & 0.3 & 0.5 \\
\hline Late vaccinationt: & & & & & & \\
\hline No immunity & 0 & 0 & 0 & 0 & 0 & 0 \\
\hline Pre-existing immunity & 0 & 0 & 0 & 0 & 0.3 & 0.5 \\
\hline $\begin{array}{l}\text { *Before peak of pandemic. } \\
\dagger \text { Peak of pandemic. }\end{array}$ & & & & & & \\
\hline
\end{tabular}


Table 4 | Healthcare resource use, unit costs, probability of use, production loss per day, and length of absence due to illness, price level 2008

\begin{tabular}{|c|c|c|c|c|c|c|}
\hline \multirow[b]{2}{*}{ Variables } & \multicolumn{2}{|c|}{ Germany } & \multicolumn{2}{|c|}{ Netherlands } & \multicolumn{2}{|c|}{ United Kingdom } \\
\hline & Unit cost $(€)$ & $\begin{array}{l}\text { Resource units or } \\
\text { probability of use }\end{array}$ & Unit cost $(€)$ & $\begin{array}{l}\text { Resource units or } \\
\text { probability of use }\end{array}$ & Unit cost $(€)$ & $\begin{array}{l}\text { Resource units or } \\
\text { probability of use }\end{array}$ \\
\hline \multicolumn{7}{|l|}{ Direct costs: } \\
\hline $\begin{array}{l}\text { Vaccine and administration } \\
\text { costs }\end{array}$ & $16^{*} \dagger$ & 2 doses & $16^{*} \dagger$ & 2 doses & $16^{*} \dagger$ & 2 doses \\
\hline $\begin{array}{l}\text { Visits to doctor if influenza-like } \\
\text { illness }\end{array}$ & $9.70^{41}$ & $24 \%{ }^{13}$ & $21.80^{24}$ & $24 \%{ }^{13}$ & $44.40 \ddagger$ & $24 \%{ }^{13}$ \\
\hline $\begin{array}{l}\text { Antibiotics prescribed by } \\
\text { doctor }\end{array}$ & $20.50^{41}$ & $17.5 \%{ }^{41}$ & $6.901^{42}$ & $20 \%{ }^{42}$ & $9.60^{43}$ & $20 \%{ }^{42}$ \\
\hline $\begin{array}{l}\text { Oseltamivir (Tamiflu) } \\
\text { prescribed by doctor* }\end{array}$ & $21^{44}$ & Treatment course & $21^{44}$ & Treatment course & $21^{44}$ & Treatment course \\
\hline $\begin{array}{l}\text { Over-the-counter drugs for } \\
\text { influenza-like illness }\end{array}$ & $6^{42}$ & $80 \%{ }^{41}$ & $6.30^{42}$ & $80 \%{ }^{42}$ & $7.30^{43}$ & $80 \%{ }^{42}$ \\
\hline Episode of hospital stay & $4075^{41}$ & - & $3398^{24}$ & - & $2892 \ddagger$ & - \\
\hline \multicolumn{7}{|l|}{ Production losses: } \\
\hline Per day, ages $20-40$ & $233^{41}$ & - & $213^{42}$ & - & $118 \ddagger$ & - \\
\hline Per day, ages 40-65 & $233^{41}$ & - & $255^{42}$ & - & $132 \ddagger$ & - \\
\hline Days of absence, all ages & - & $2.16^{41}$ & - & $3.25^{42}$ & - & $2.9 \ddagger$ \\
\hline
\end{tabular}

Dutch data are used when country specific data are missing. Prices updated to 2008 using country specific consumer price index.

*Per dose, including costs of administering drug.

†Assumed the same for all three countries.

‡Personal communication, K Tolley, 2008. 
Table 5| One year losses in quality of life due to influenza infection

\begin{tabular}{lccc} 
& & \multicolumn{2}{c}{ Pandemic influenza } \\
\cline { 3 - 4 } Infection status & Seasonal influenza & Adults & Young people (<18 years) \\
Influenza infection & $0.01^{28}$ & $0.0074^{7}$ & $0.0082^{7}$ \\
\hline Influenza infection with admission to hospital & $0.0217^{7}$ & $0.016^{7}$ & $0.018^{7}$
\end{tabular}

Value of 1 represents death and value of 0 represents full health. 
Table 6| Results for cost effectiveness: vaccination costs, net direct costs, net total costs, quality adjusted life years (QALYs) gained, and incremental cost effectiveness ratios (ICERs) comparing vaccination strategy with no vaccination for Germany, Netherlands, and United Kingdom; price level 2008

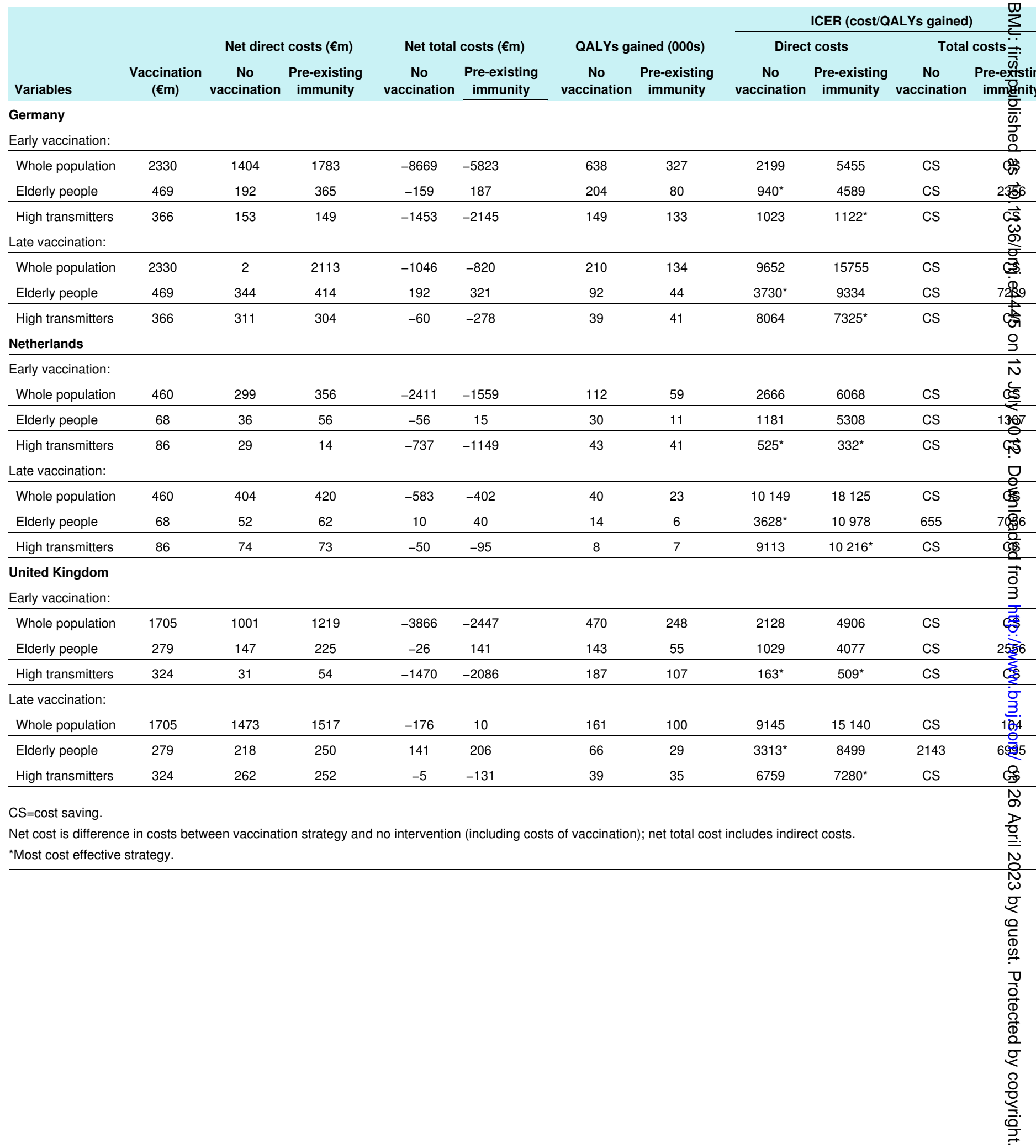


Table 7 | Overview of most cost effective vaccination strategy by country, vaccine availability, and immunity scenario

\begin{tabular}{lll} 
Variables & & \multicolumn{1}{c}{ No immunity } \\
Germany: & & Pre-existing immunity \\
\hline Early vaccination & Elderly people & High transmitters \\
\hline Late vaccination & Elderly people & High transmitters \\
\hline Netherlands: & & \\
\hline Early vaccination & High transmitters & High transmitters \\
\hline Late vaccination & Elderly people & High transmitters \\
\hline United Kingdom: & & \\
\hline Early vaccination & High transmitters & High transmitters \\
\hline Late vaccination & Elderly people & High transmitters \\
\hline
\end{tabular}

Cost effectiveness is calculated using direct costs only. See table 6 for full results. Scenarios considered are no vaccination, vaccination of whole population, vaccination of elderly people, and vaccination of high transmitters (young people aged 5-19 years). Table 2 provides an overview of scenarios. 
Table 8 | Overview of sensitivity analyses of most cost effective vaccination strategy (direct costs) by country, vaccine availability, and immunity scenario

\begin{tabular}{|c|c|c|c|c|c|c|}
\hline \multirow[b]{2}{*}{ Vaccination strategy } & \multicolumn{2}{|c|}{ Germany } & \multicolumn{2}{|c|}{ Netherlands } & \multicolumn{2}{|c|}{ United Kingdom } \\
\hline & No immunity & $\begin{array}{l}\text { Pre-existing } \\
\text { immunity }\end{array}$ & No immunity & $\begin{array}{l}\text { Pre-existing } \\
\text { immunity }\end{array}$ & No immunity & $\begin{array}{l}\text { Pre-existing } \\
\text { immunity }\end{array}$ \\
\hline \multicolumn{7}{|l|}{ High vaccine cost: } \\
\hline Early vaccination & Elderly people & High transmitters & High transmitters & High transmitters & High transmitters & High transmitters \\
\hline Late vaccination & Elderly people & High transmitters & Elderly people & High transmitters & Elderly people & High transmitters \\
\hline \multicolumn{7}{|l|}{ No discounting: } \\
\hline Early vaccination & Elderly people & High transmitters & High transmitters & High transmitters & High transmitters & High transmitters \\
\hline Late vaccination & Elderly people & High transmitters & Elderly & High transmitters & Elderly people & High transmitters \\
\hline \multicolumn{7}{|l|}{ High transmissibility: } \\
\hline Early vaccination & Elderly people & High transmitters & High transmitters & High transmitters & High transmitters & High transmitters \\
\hline Late vaccination & Elderly people & Elderly people & Elderly people & Elderly people & Elderly people & Elderly people \\
\hline \multicolumn{7}{|l|}{ Low transmissibility: } \\
\hline Early vaccination & High transmitters & High transmitters & High transmitters & High transmitters & High transmitters* & High transmitters* \\
\hline Late vaccination & Elderly people & High transmitters & Elderly people & High transmitters & Elderly people & High transmitters \\
\hline \multicolumn{7}{|l|}{$\begin{array}{l}\text { Low vaccination } \\
\text { coverage: }\end{array}$} \\
\hline Early vaccination & Whole population & High transmitters & High transmitters & High transmitters & High transmitters & High transmitters \\
\hline Late vaccination & Elderly people & High transmitters & Elderly people & High transmitters & Elderly people & High transmitters \\
\hline \multicolumn{7}{|l|}{$\begin{array}{l}\text { High pre-existing } \\
\text { immunity: }\end{array}$} \\
\hline Early vaccination & NA & High transmitters & NA & High transmitters & NA & High transmitters \\
\hline Late vaccination & NA & High transmitters & NA & High transmitters & NA & High transmitters \\
\hline
\end{tabular}

$\mathrm{NA}=$ not applicable

Assumptions underlying sensitivity analyses are given in the methods. Supplementary tables A.4 to A.6 show the full sensitive results, including interactions between factors, incremental cost effectiveness ratios, and scenarios with indirect costs. Cost effectiveness is calculated excluding indirect costs. Scenarios are: vaccination of whole population, vaccination of elderly people, and vaccination of high transmitters-all compared with no vaccination. See table 2 for overview of scenarios.

${ }^{*}$ Cost saving strategy. 
Figure
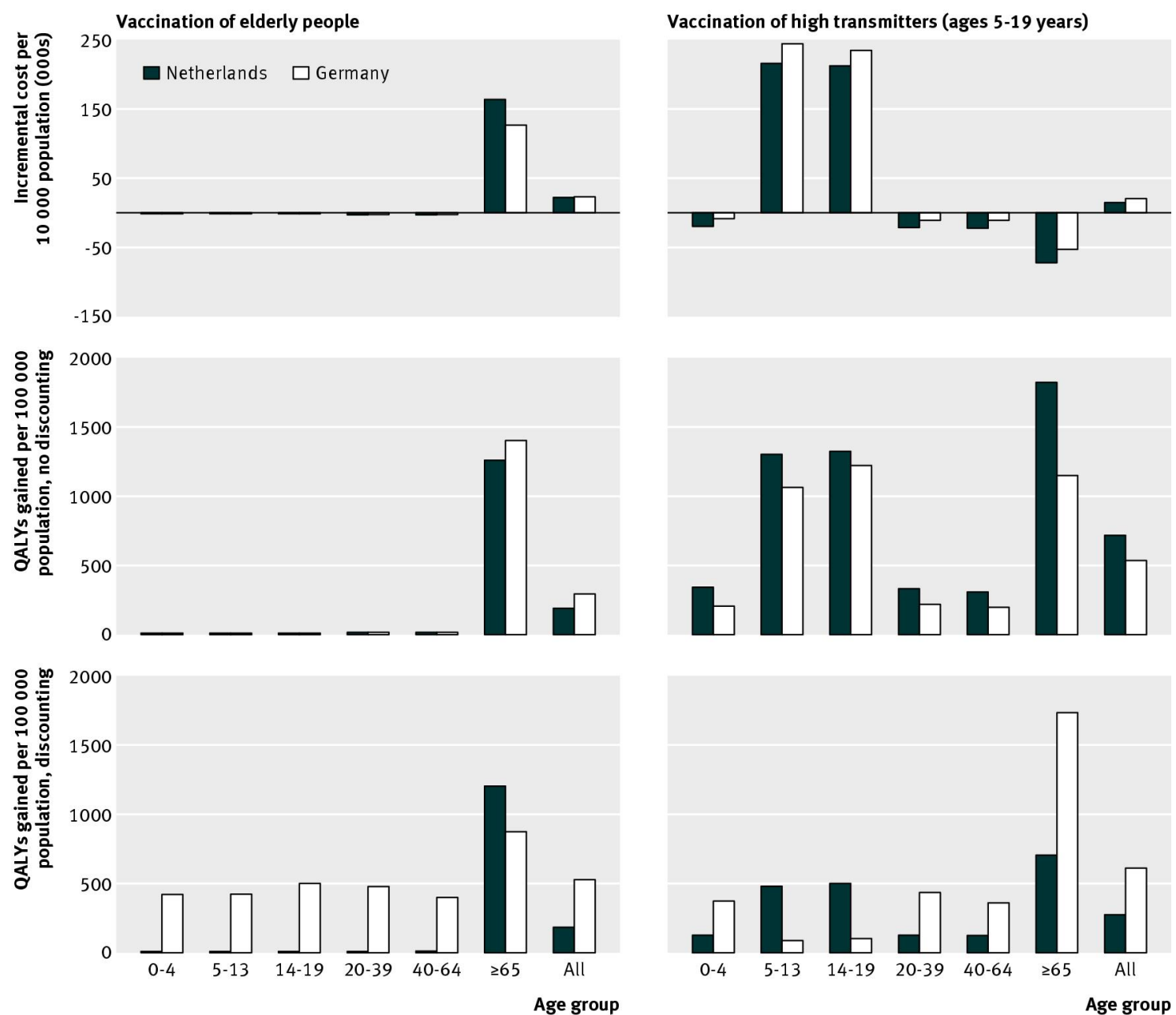

Incremental costs per 10000 population and quality adjusted life years (QALYs) gained per 100000 population, comparing vaccinations strategies for elderly people and high transmitters (vaccine available early in pandemic, no immunity present) for the Netherlands and Germany (direct healthcare costs $(€)$, discounted (Germany $5 \%$, Netherlands $1.5 \%$ ) and without discounting, price level 2008) 